\title{
Prognostic relevance of TTF-1 expression in stage I adenocarcinoma
}

\author{
Chao Zhou' ${ }^{1}$, Jikai Zhao ${ }^{2}$, Jinchen Shao ${ }^{2}$ and Wentao $\mathrm{Li}^{1}$ \\ ${ }^{1}$ Department of Thoracic Surgery, Shanghai Chest Hospital, Shanghai Jiao Tong University, Shanghai 200030, China \\ ${ }^{2}$ Department of Pathology, Shanghai Chest Hospital, Shanghai Jiao Tong University, Shanghai 200030, China
}

Correspondence to: Wentao Li, email: leetootoo@hotmail.com

Keywords: thyroid transcription factor-1; lung adenocarcinoma; subtype; adjuvant chemotherapy; prognosis

Received: June 27, $2017 \quad$ Accepted: October 28, $2017 \quad$ Published: November 18, 2017

Copyright: Zhou et al. This is an open-access article distributed under the terms of the Creative Commons Attribution License 3.0 (CC BY 3.0), which permits unrestricted use, distribution, and reproduction in any medium, provided the original author and source are credited.

\section{ABSTRACT}

Tyroid transcription factor-1 (TTF-1) motivates the differentiation and development of bronchioloalveolar cells. The association of TTF-1 expression with prognosis in stage I adenocarcinoma is unclear. This study enrolled patients with resected stage I pulmonary adenocarcinoma who had TTF-1 immunostaining. All the corresponding clinicopathologic data including sex, age, smoking history, pathologic T stage, pathologic disease stage, surgical procedure, subtypes, follow-up records and adjuvant chemotherapy were investigated. Totally, 126 adenocarcinomas with TTF-1 - and 2687 adenocarcinomas with TTF-1+ were subjected to the study. Among adenocarcinomas with TTF-1-, the major subtype was acinar-predominant adenocarcinomas, followed by invasive mucinous and papillary subtypes while acinar, papillary and minimally invasive adenocarcinoma were in the majority among adenocarcinomas with TTF-1+. The status of TTF-1 expression was not a significant factor for relapse-free survival (RFS) and overall survival (OS). Furthermore, there was no survival difference between the two groups (RFS: $p=0.2474 ;$ OS: $p=0.1480$ ). When confined to stage IB adenocarcinomas with TTF-1-, whether received adjuvant chemotherapy made no difference to RFS and OS (RFS: $p=0.2707$; OS: $p=1.000$ ), as was the case in stage IB adenocarcinomas with TTF-1+ (RFS: $p=0.9161$; OS: $p=0.1100)$. Within follow-up period, there was significant difference in postrecurrence survival (PRS) for TTF-1- patients compared with those TTF-1+ patients (Log-rank $p=0.0113$ ). However, regarding to the recurrence site, there was no difference between TTF-1 - patients and TTF-1 + patients in patients with stage I adenocarcinoma $(p=0.771)$ In conclusion, there is no significant difference in RFS and $0 S$ between TTF-1 - group and TTF-1+ group, but TTF-1 negative adenocarcinoma has significantly worse PFS in patients with stage I adenocarcinoma. Moreover, chemotherapeutic efficacy between TTF-1+ and TTF-1- stage IB adenocarcinomas did not differ.

\section{INTRODUCTION}

Lung cancer remains the leading cause of cancer death worldwide in recent years [1]. Lung adenocarcinoma, accounting for nearly $50 \%$ of all lung cancer, is the most common histologic subtype and the morbidity rate is increasing year by year [2]. Prognosis of lung adenocarcinoma has raised concern by the International Association for the Study of Lung Cancer (IASLC)/
American Thoracic Society (ATS)/European Respiratory Society (ERS) in 2011 [3].

Thyroid transcription factor-1 (TTF-1), known as a member of homeodomain-containing nuclear transcriptional protein of the $\mathrm{Nkx} 2$ gene family, motivates the differentiation and development of bronchioloalveolar cells [4]. In normal adult lung, the expression of TTF-1 is confined mainly to terminal respiratory units $[5,6]$. Currently, TTF-1, detecting in the immunohistochemistry 
panel, acts as an important prognostic marker for diagnosis of lung adenocarcinoma, which was also proposed by IASLC/ATS/ERS [3, 7-9]. Winslow et al. found increase of TTF-1 expression revealed a better outcome while reduced expression would enhance the ability of tumor seeding and metastasis [10]. Previous studies have demonstrated that positive TTF-1 expression (TTF-1+) is a significant prognostic factor for better outcome in pulmonary adenocarcinoma [11-14]. And some studies also revealed the relationship between TTF-1 expression and gene mutations that a higher epidermal growth factor receptor (EGFR) mutation rate would be found in TTF-1 positive adenocarcinoma $[15,16]$. However, the patients in their researches were commonly with advanced diseases and the chemotherapeutic effect between TTF-1 positive adenocarcinoma and TTF-1 negative adenocarcinoma, especially in early-stage lung adenocarcinoma is unknown. Thus, we undertook an investigation of TTF1 expression in stage I adenocarcinoma and compared the chemotherapeutic effect between TTF-1 positive adenocarcinoma and TTF-1 negative adenocarcinoma in pathological stage IB patients.

\section{RESULTS}

\section{Clinicopathologic characteristics}

In total, 2813 stage I patients including 126 adenocarcinomas with negative TTF-1 expression and 2687 adenocarcinomas with positive TTF-1 expression were subjected to the study. There were 1527 (54.3\%) men and $1286(45.7 \%)$ women in the series, with the mean age of 60.7 years (from 24 to 85 years).

The comparative clinicopathologic parameters between adenocarcinomas with positive TTF-1 expression and adenocarcinomas with negative TTF-1 expression are shown in Table 1. Sex, age, smoking history, T stage, disease stage, surgical procedure, subtypes and the treatment of adjuvant chemotherapy were analyzed. The characteristics between the two groups were similar.

As for the relationship between TTF-1 expression and subtypes, among adenocarcinomas with negative TTF-1 expression, the major subtype was acinar-predominant adenocarcinomas $(28.6 \%)$, followed by invasive mucinous $(27.8 \%)$ and papillary (18.3\%) subtypes while acinar $(40.9 \%)$, papillary $(31.7 \%)$ and minimally invasive adenocarcinoma $(7.5 \%)$ were in the majority among adenocarcinomas with TTF-1+ (Table 1).

\section{Survival analysis}

Univariable analysis revealed that age, disease stage, tumor size, $\mathrm{T}$ stage and surgical procedure were all significant factors for relapse-free survival (RFS) and overall survival (OS) (Table 2) while age and surgical procedure were still significant factors for RFS and OS in multivariable analysis (Table 3). The status of TTF-1 expression was not a significant factor for RFS and OS.

In stage I adenocarcinomas, there was no survival difference between the two groups (RFS: Log-rank $p=0.2474$; OS: Log-rank $p=0.1480$, Figure 1A and 1B). When confined to stage IB adenocarcinomas with TTF$1-$, whether received adjuvant chemotherapy made no difference to RFS and OS (RFS: Log-rank $p=0.2707$; OS: Log-rank $p=1.000$, Figure 1C and 1D), as was the case in stage IB adenocarcinomas with TTF-1+ (RFS: Log-rank $p=0.9161 ;$ OS: Log-rank $p=0.1100$, Figure $1 \mathrm{E}$ and $1 \mathrm{~F})$.

Within follow-up period, a total of $13(10.3 \%)$ TTF1 - patients and $163(6.1 \%) \mathrm{TTF}-1+$ patients experienced a relapse. There was significant difference in postrecurrence survival (PRS) for TTF-1- patients compared with those TTF-1+ patients (Log-rank $p=0.0113$, Figure 2). However, regarding to the recurrence site, there was no difference between TTF-1- patients and TTF-1+ patients in patients with stage I adenocarcinoma $(30.8 \%$ versus $36.8 \%, p=0.771$ ).

\section{DISCUSSION}

As IASLC/ATS/ERS emphasized in the new classification in 2011, immunohistochemical (including TTF-1), combined with histochemical and molecular studies were suggested to determine the specific type of lung cancer. Furthermore, TTF-1, acted as a pneumocyte marker, was considered to be the single best marker for lung adenocarcinoma and had the added value to distinguish primary lung cancer $[3,5,17,18]$. In our study, we reviewed 2813 stage I patients including 126 adenocarcinomas with TTF-1+ and 2687 adenocarcinomas with TTF-1- to have a comprehensive understanding of the clinicopathologic features, patterns of recurrence, chemotherapeutic efficacy and the prognosis associated with TTF-1 expression. Zhang and his colleagues reported that patients with TTF-1- were inclined to be male smokers with larger tumor size and more advanced disease stage. Besides, patients with TTF-1- tended to have multiple metastases when they experienced a relapse [16]. Nevertheless, we found that in early stage adenocarcinomas, the characteristics between TTF-1+ group and TTF-1- group were similar, even the patterns of recurrence $(p=0.771)$. The reason may be because the more advanced disease stage was, the less TTF-1 would be expressed, and all the patients in our study were stage I patients while more patients with advanced stage in theirs.

In this study, TTF-1- adenocarcinomas most commonly appeared in acinar-predominant subtype, followed by followed by invasive mucinous and papillary subtypes, which was basically in accordance with previous studies [16, 19]. As Saad et al [20]. revealed, invasive mucinous adenocarcinoma was mostly negative for TTF-1, which was in consistence with our results. Interestingly, the 2011 IASLC/ATS/ERS classification indicated that 
Table 1: Clinicopathologic characteristics

\begin{tabular}{|c|c|c|c|}
\hline Variable & TTF-1- $(n=126)$ & TTF-1+ $(n=2687)$ & $P$ \\
\hline Sex & & & 0.769 \\
\hline Male & 70 & 1457 & \\
\hline Female & 56 & 1230 & \\
\hline Age & & & 0.513 \\
\hline$<65$ & 79 & 1761 & \\
\hline$\geq 65$ & 47 & 926 & \\
\hline Smoking history & & & 0.436 \\
\hline Smoker & 8 & 223 & \\
\hline Non-smoker & 116 & 2464 & \\
\hline T stage & & & 0.486 \\
\hline 1a & 69 & 1483 & \\
\hline $1 b$ & 26 & 667 & \\
\hline $2 \mathrm{a}$ & 31 & 537 & \\
\hline Stage & & & 0.238 \\
\hline IA & 95 & 2151 & \\
\hline IB & 31 & 536 & \\
\hline Surgical procedure & & & 0.928 \\
\hline Lobectomy & 109 & 2332 & \\
\hline Wedge resection & 17 & 355 & \\
\hline Subtypes & & & 0.521 \\
\hline AIS & 3 & 57 & \\
\hline MIA & 3 & 201 & \\
\hline Lepidic & 4 & 197 & \\
\hline Acinar & 36 & 1098 & \\
\hline Papillary & 23 & 851 & \\
\hline Micropapillary & 1 & 32 & \\
\hline Solid & 19 & 151 & \\
\hline Invasive mucinous & 35 & 94 & \\
\hline Others & 2 & 6 & \\
\hline Adjuvant chemotherapy & & & 0.384 \\
\hline Yes & 26 & 2214 & \\
\hline No & 100 & 473 & \\
\hline
\end{tabular}

Abbreviations: TTF-1, thyroid transcription factor 1; AIS, adenocarcinoma in situ; MIA, minimally invasive adenocarcinoma.

all the nonmucinous adenocarcinoma in situ (AIS), minimally invasive adenocarcinoma (MIA) and lepidic adenocarcinoma were negative for TTF-1. But in our series, we found several patients were TTF-1+ (3 AISs, 3 MIAs, 4 lepidic adenocarcinomas), which needed further study.

Numerous studies have investigated the relationship between TTF-1 expression and prognosis, most of which demonstrated that positive TTF-1 expression was associated with better survival [11, 13, 21-23]. But in our study, there was no difference between TTF-1- group and TTF-1+ group in RFS (Log-rank $p=0.2474$ ) and OS (Log-rank $p=0.1480)$, which was in accordance with Pelosi [24]. Moreover, we further investigated chemotherapeutic efficacy of stage IB adenocarcinomas between TTF-1- group and TTF-1+ group. Whether received adjuvant chemotherapy made no difference to RFS and OS (RFS: Log-rank $p=0.2707$; OS: Logrank $p=1.000$, Figure $1 \mathrm{C}$ and $1 \mathrm{D})$, as was the case in stage IB adenocarcinomas with TTF-1+ (RFS: Log-rank $p=0.9161$; OS: Log-rank $p=0.1100$, Figure $1 \mathrm{E}$ and $1 \mathrm{~F})$. 
Table 2: Univariable analyses for RFS and OS in our study

\begin{tabular}{|c|c|c|c|c|c|c|}
\hline \multirow{2}{*}{ Variable } & \multicolumn{3}{|c|}{ RFS } & \multicolumn{3}{|c|}{ OS } \\
\hline & HR & $95 \% \mathrm{CI}$ & $p$ & HR & $95 \% \mathrm{CI}$ & $p$ \\
\hline Age, yrs & 1.915 & 1.425 to 2.575 & $<0.001$ & 2.181 & 1.432 to 3.321 & $<0.001$ \\
\hline Sex & 1.180 & 0.878 to 1.585 & 0.273 & 1.269 & 0.833 to 1.934 & 0.267 \\
\hline Smoking history & 0.749 & 0.434 to 1.295 & 0.301 & 0.930 & 0.448 to 1.932 & 0.846 \\
\hline Stage & 3.396 & 2.525 to 4.567 & $<0.001$ & 2.520 & 1.643 to 3.866 & $<0.001$ \\
\hline T-size, cm & 2.515 & 1.951 to 3.241 & $<0.001$ & 1.991 & 1.407 to 2.818 & $<0.001$ \\
\hline T Stage & 2.232 & 1.861 to 2.677 & $<0.001$ & 1.910 & 1.481 to 2.462 & $<0.001$ \\
\hline Subtypes & 0.988 & 0.945 to 1.034 & 0.614 & 0.990 & 0.929 to 1.055 & 0.754 \\
\hline TTF-1, +/- & 0.730 & 0.414 to 1.287 & 0.277 & 0.601 & 0.299 to 1.207 & 0.152 \\
\hline Surgical procedure & 0.402 & 0.274 to 0.592 & $<0.001$ & 0.282 & 0.170 to 0.467 & $<0.001$ \\
\hline Adjuvant chemotherapy & 2.373 & 1.743 to 3.231 & $<0.001$ & 1.332 & 0.820 to 2.161 & 0.246 \\
\hline
\end{tabular}

Abbreviations: RFS, relapse-free survival; OS, overall survival; HR, hazard ratio.

Table 3: Multivariable analyses for RFS and OS in our study

\begin{tabular}{|c|c|c|c|c|c|c|}
\hline \multirow{2}{*}{ Variable } & \multicolumn{3}{|c|}{ RFS } & \multicolumn{3}{|c|}{ OS } \\
\hline & HR & $95 \% \mathrm{CI}$ & $p$ & HR & $95 \% \mathrm{CI}$ & $p$ \\
\hline Age, yrs & 1.644 & 1.206 to 2.240 & 0.002 & 1.687 & 1.094 to 2.062 & 0.018 \\
\hline Stage & 1.858 & 0.835 to 4.134 & 0.129 & 0.934 & 0.310 to 2.817 & 0.903 \\
\hline T-size, cm & 2.006 & 1.384 to 2.908 & $<0.001$ & 1.464 & 0.878 to 2.440 & 0.144 \\
\hline T Stage & 1.139 & 0.653 to 1.984 & 0.647 & 1.723 & 0.803 to 3.697 & 0.162 \\
\hline Surgical procedure & 0.377 & 0.252 to 0.563 & $<0.001$ & 0.272 & 0.160 to 0.464 & $<0.001$ \\
\hline Adjuvant chemotherapy & 1.384 & 0.969 to 1.977 & 0.074 & & & \\
\hline
\end{tabular}

Abbreviations: RFS, relapse-free survival; OS, overall survival; HR, hazard ratio.

The reason was as follows: it is generally known that on the promise of complete resection, the later pathological stage is, the poorer prognosis would be. All the patients in our study was stage I adenocarcinomas while more advanced disease made up the majority in other studies.

In our series, TTF-1 was not recognized as an independent prognostic factor, neither in univariable analysis nor in multivariable analysis. Disease stage may still be the major reason. Within the follow-up period, a total of 13 (10.3\%) TTF-1- patients and 163 (6.1\%) TTF$1+$ patients experienced a relapse. There was significant difference in post-recurrence survival (PRS) for TTF-1patients compared with those TTF-1+ patients (Log-rank $p=0.0113$, Figure 2). The results was basically in line with zhang and his colleagues [16], who also found pathologic stage, TTF-1 expression and the use of EGFR TKIs as independent predictors of post-recurrence survival.

In recent years, molecular researches on mutational status were carried out widely. A higher EGFR mutation rate was identified in TTF-1+ adenocarcinomas, but a fraction of TTF-1- adenocarcinomas still had EGFR mutation $[15,25,26]$. They also revealed that under the treatment of EGFR TKIs, adenocarcinomas with TTF-1+ and EGFR mutation had a better outcome and in EGFRmutated patients, those with TTF-1- adenocarcinoma had worse PFS than what those with TTF-1+ adenocarcinoma did [13]. This would be investigated in our future work.

There are several limitations in our study. First, this study is not a randomized prospective study, selection bias may exist. Second, in the subset of stage IB TTF1 - patients, the chemotherapeutic efficacy could not be shown well due to the small amount of sample size.

In summary, in patients with stage I adenocarcinoma, there is no significant difference in RFS and OS between TTF-1- group and TTF-1+ group, but TTF-1 negative adenocarcinoma has significantly worse PFS. Moreover, chemotherapeutic efficacy between TTF-1+ and TTF-1stage IB adenocarcinomas did not differ.

\section{MATERIALS AND METHODS}

From Jan. 2010 to Aug. 2015, we consecutively collected resected stage I pulmonary tumors in the Department of Thoracic Surgery, Shanghai Chest hospital. Two pathologists (J.K.Z and J.C.S.) confirmed the diagnosis 
A

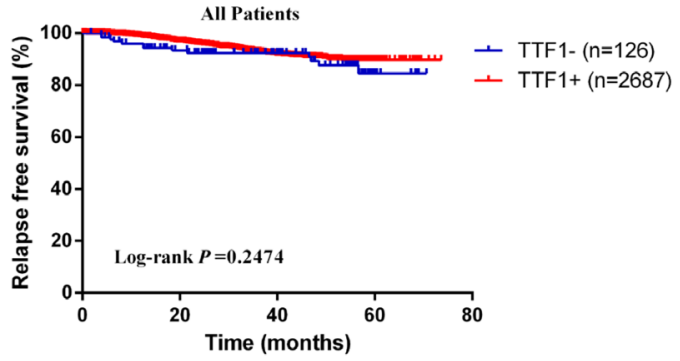

C

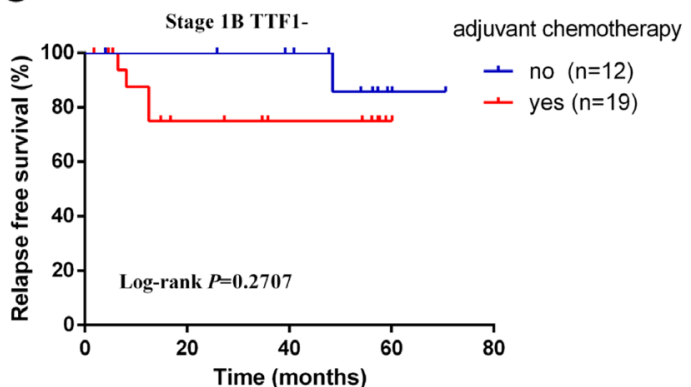

E

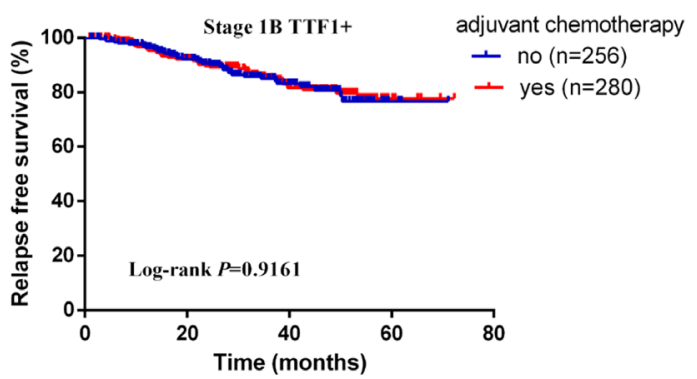

B

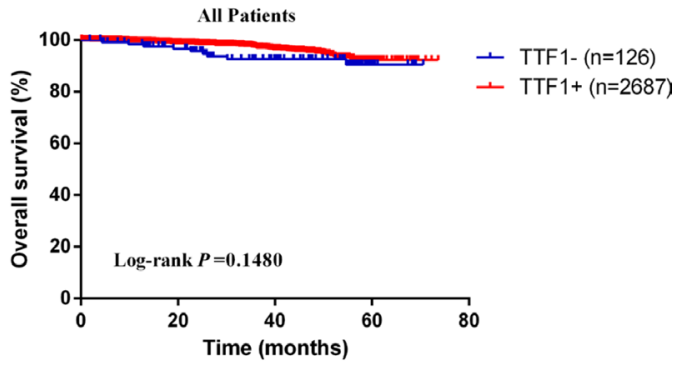

D

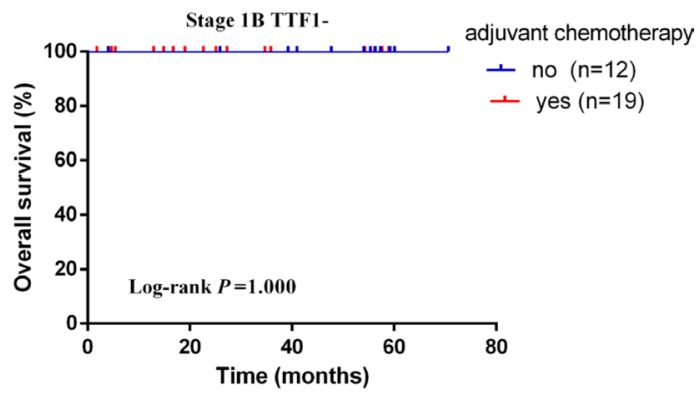

$\mathbf{F}$

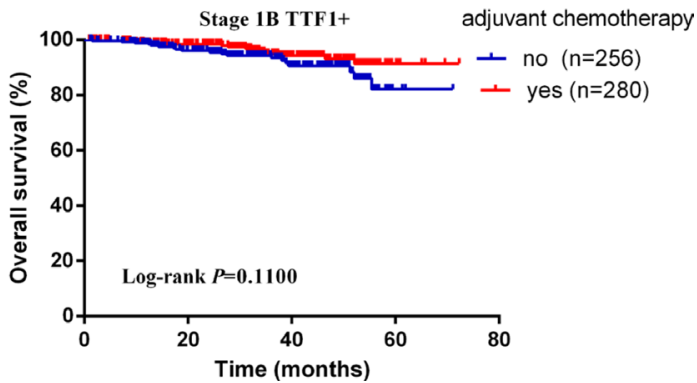

Figure 1: Kaplan-Meier survival curves for recurrence-free survival (RFS) and overall survival (OS) according to TTF-1 status. (A) RFS in all patients. (B) OS in all patients. (C) RFS in stage IB TTF-1- patients. (D) OS in stage IB TTF-1- patients. (E) RFS in stage IB TTF-1+ patients. (F) OS in stage IB TTF-1+ patients.

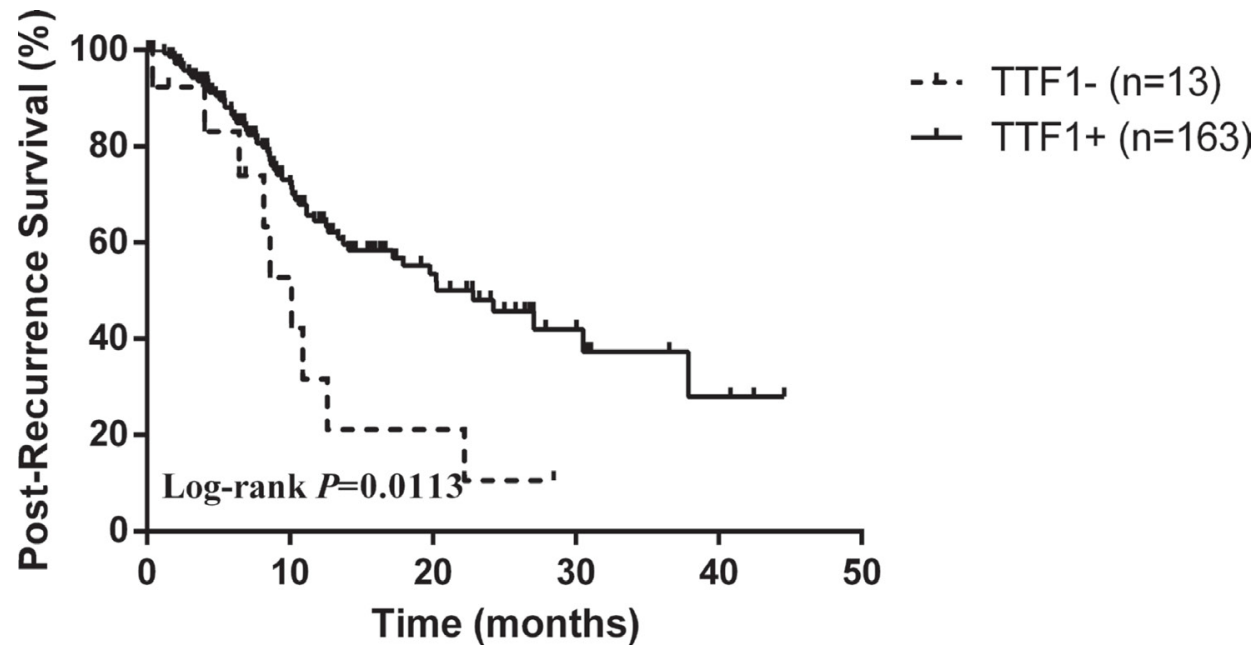

Figure 2: Kaplan-Meier survival curve for post-recurrence survival. 
by H\&E staining and immunohistochemistry biomarkers. To exclude tumor metastasis, all the patients were under routine preoperative examinations including computed tomography (CT) or enhanced thoracic computed tomography, abdominal ultrasonography, brain magnetic resonance imaging (MRI) and bone scan. Furthermore, suspicious lymph node metastasis or distant metastasis which could not be excluded by routine preoperative examinations, endobronchial ultrasound-guided transbronchial needle aspiration (EBUSTBNA) or positron emission tomography (PET)-CT scan was recommended. In our study, the inclusion criteria included:(1) patients with lung adenocarcinoma underwent R0 resection; (2) the status of TTF-1 (positive or negative) was available by immunohistochemistry test; (3) patients with complete follow-up records; (4) those with metastatic lung adenocarcinoma were excluded. Of these, we identified 2813 stage I adenocarcinomas with available clinicopathologic data and postoperative follow-up records.

Signed informed consents of patients were obtained and this study was approved by our institutional review board. All the corresponding clinicopathologic data including sex, age, smoking history, pathologic T stage, pathologic disease stage (according to AJCC TNM staging system 7th edition [17], surgical procedure, subtypes and adjuvant chemotherapy were investigated.

Immunohistochemical (IHC) staining was carried out on 4- $\mu \mathrm{m}$ formalin-fixed, paraffin-embedded tissue samples. Slides were deparaffinized and pretreated in $3 \%$ hydrogen peroxide and ethylenediaminetetraacetic acid successively. TTF-1 (1:100, 8G7G3/1, DAKO, glostrop, Denmark) was used at a 1:200 dilution. Then, the slides were washed in Tris- $\mathrm{HCl}$ and affected using horseradish peroxidase-conjugated anti-rabbit horseradish peroxidase-conjugated anti-rabbit EnVision+ kit (DAKO). Hematoxylin was applied to counterstain all the slides. Sole nuclear staining was considered TTF-1 positive.

\section{Statistical analysis}

SPSS 19.0 software package (SPSS Inc, Chicago, IL) was used to analyze the clinicopathologic data and distributions of relapse-free survival (RFS), overall survival (OS) and post-recurrence survival (PRS) were established by Prism 5 (Graph Pad Software Inc., La Jolla, CA), using the Kaplan-Meier method, and the comparisons between two categories was employed by the log-rank test. A $p$ value less than 0.05 was considered as statistically significant.

Cox regression was adopted to perform univariable and multivariable analyses. The end point was recognized as event occurrence (relapse for RFS while death for OS). All the correlative clinicopathologic characteristics were enrolled into univariable analysis one by one, with the method "Enter". Moreover, all the correlative clinicopathologic characteristics were brought into multivariable analysis together when $p$ value had significance $(<0.05)$ in univariable analysis.

\section{Abbreviations}

TTF-1: thyroid transcription factor-1; RFS: relapsefree survival; OS: overall survival; PRS: post-recurrence survival; IASLC: the International Association for the Study of Lung Cancer; ATS: American Thoracic Society; ERS: European Respiratory Society; EGFR: epidermal growth factor receptor; AIS: adenocarcinoma in situ; MIA: minimally invasive adenocarcinoma; CT: computed tomography; MRI: magnetic resonance imaging; IHC: Immunohistochemical; EBUS-TBNA: endobronchial ultrasound-guided transbronchial needle aspiration; PET: positron emission tomography.

\section{Ethics statement}

This study was conducted in line with the Helsinki Declaration, and was approved by the Institutional Review Board of the Shanghai Chest Hospital. Written informed consent was obtained from each patient. The experimental protocol of this study was performed strictly in accordance to the guidelines.

\section{Author contributions}

C.Z. and W.T.L. designed this study, performed experiments and wrote the main manuscript. C.Z. J.C.S. and J.K.Z. collected the clinical data, tumor samples and performed experiments. C.Z. and WT.L. designed and directed the overall project. All authors reviewed the manuscript.

\section{REFERENCES}

1. Jemal A, Bray F, Center MM, Ferlay J, Ward E, Forman D. Global cancer statistics. CA Cancer J Clin. 2011; 61:69-90.

2. Devesa SS, Bray F, Vizcaino AP, Parkin DM. International lung cancer trends by histologic type: male: female differences diminishing and adenocarcinoma rates rising. Int J Cancer. 2005; 117:294-299.

3. Travis WD, Brambilla E, Noguchi M, Nicholson AG, Geisinger KR, Yatabe Y, Beer DG, Powell CA, Riely GJ, Van Schil PE, Garg K, John H. M. Austin, Asamura H, et al. IASLC/ATS/ERS international multidisciplinary classifcation of lung adenocarcinoma. J Thorac Oncol. 2011; 6:244-285.

4. Maeda Y, Dave V, Whitsett JA. Transcriptional control of lung morphogenesis. Physiol Rev. 2007; 87:219-244.

5. Yatabe Y, Mitsudomi T, Takahashi T. TTF-1 expression in pulmonary adenocarcinomas. Am J Surg Pathol. 2002; 26:767-773.

6. Zhang L, Whitsett JA, Stripp BR. Regulation of Clara cell secretory protein gene transcription by thyroid transcription factor-Biochimica et Biophysica Acta (BBA)-Gene Structure and Expression. 1997; 1350:359-367. 
7. Stenhouse G, Fyfe N, King G, Chapman A, Kerr KM. Thyroid transcription factor-1 in pulmonary adenocarcinoma. J Clin Pathol. 2004; 57:383-387.

8. Kaufmann $\mathrm{O}$, Dietel $\mathrm{M}$. Thyroid transcription factor-1 is the superior immunohistochemical marker for pulmonary adenocarcinomas and large cell carcinomas compared to surfactant proteins A and B. Histopathology. 2000; 36:8-16.

9. Chang YL, Lee YC, Liao WY, Wu CT. The utility and limitation of thyroid transcription factor-1 protein in primary and metastatic pulmonary neoplasms. Lung Cancer. 2004; 44:149-157.

10. Winslow MM, Dayton TL, Verhaak RG, Kim-Kiselak C, Snyder EL, Feldser DM, Hubbard DD, DuPage MJ, Whittaker CA, Hoersch S, Yoon S, Crowley D, Bronson RT, et al. Suppression of lung adenocarcinoma progression by Nkx2-1. Nature. 2011; 473:101-104.

11. Barletta JA, Perner S, Iafrate AJ, Yeap BY, Weir BA, Johnson LA, Johnson BE, Meyerson M, Rubin MA, Travis WD, Loda M, Chirieac LR. Clinical significance of TTF-1 protein expression and TTF-1 gene amplification in lung adenocarcinoma. J Cell Mol Med. 2009; 13:1977-1986.

12. Barlesi F, Pinot D, Legoffic A, Doddoli C, Chetaille B, Torre JP, Astoul P. Positive thyroid transcription factor 1 staining strongly correlates with survival of patients with adenocarcinoma of the lung. Br J Cancer. 2005; 93:450-452.

13. Chung KP, Huang YT, Chang YL, Yu CJ, Yang CH, Chang YC, Shih JY, Yang PC. Clinical significance of thyroid transcription factor-1 in advanced lung adenocarcinoma under epidermal growth factor receptor tyrosine kinase inhibitor treatment. Chest. 2012; 141:420-428.

14. Anagnostou VK, Syrigos KN, Bepler G, Homer RJ, Rimm DL. Thyroid transcription factor 1 is an independent prognostic factor for patients with stage I lung adenocarcinoma. J Clin Oncol. 2009; 27:271-278.

15. Yatabe Y, Kosaka T, Takahashi T, Mitsudomi T. EGFR mutation is specific for terminal respiratory unit type adenocarcinoma. Am J Surg Pathol. 2005; 29:633-639.

16. Zhang Y, Wang R, Li Y, Pan Y, Hu H, Zhang Y, Li H, Shen L, Yu Y, Sun Y, Chen H. Negative thyroid transcription factor 1 expression defines an unfavorable subgroup of lung adenocarcinomas. J Thorac Oncol. 2015; 10:1444-1450.

17. Edge SB, Compton CC. The American Joint Committee on Cancer: the 7th edition of the AJCC cancer staging manual and the future of TNM. Ann Surg Oncol. 2010; 17:1471-1474.
18. Lau SK, Luthringer DJ, Eisen RN. Thyroid transcription factor-1: a review. Appl Immunohistochem Mol Morphol. 2002; 10:97-102.

19. Kadota K, Nitadori JI, Sarkaria IS, Sima CS, Jia XY, Yoshizawa A, Rusch VW, Travis WD, Adusumilli PS. Thyroid transcription factor-1 expression is an independent predictor of recurrence and correlates with the IASLC/ATS/ERS histologic classification in patients with stage I lung adenocarcinoma. Cancer. 2013; 119:931-938.

20. Saad RS, Cho P, Silverman JF, Liu YL. Usefulness of Cdx2 in separating mucinous bronchioloalveolar adenocarcinoma of the lung from metastatic mucinous colorectal adenocarcinoma. Am J Clin Pathol. 2004; 122:421-427.

21. Solis LM, Behrens C, Raso MG, Lin HY, Kadara H, Yuan P, Galindo H, Tang X, Lee JJ, Kalhor N, Wistuba II, Moran CA. Histologic patterns and molecular characteristics of lung adenocarcinoma associated with clinical outcome. Cancer. 2012; 118:2889-2899.

22. Tang X, Kadara H, Behrens C, Liu DD, Xiao Y, Rice D, Gazdar AF, Fujimoto J, Moran C, Varella-Garcia M, Lee JJ, Hong WK, Wistuba II. Abnormalities of the TTF-1 lineage-specific oncogene in NSCLC: implications in lung cancer pathogenesis and prognosis. Clin Cancer Res. 2011; 17:2434-2443.

23. Li X, Wan L, Shen H, Geng J, Nie J, Wang G, Jia N, Dai $\mathrm{M}$, Bai X. Thyroid transcription factor-1 amplification and expressions in lung adenocarcinoma tissues and pleural effusions predict patient survival and prognosis. J Thorac Oncol. 2012; 7:76-84.

24. Pelosi G, Fraggetta F, Pasini F, Maisonneuve P, Sonzogni A, Iannucci A, Terzi A, Bresaola E, Valduga F, Lupo C, Viale G. Immunoreactivity for thyroid transcription factor-1 in stage I non-small cell carcinomas of the lung. Am J Surg Pathol. 2001; 25:363-372.

25. Shanzhi W, Yiping H, Ling H, Jianming Z, Qiang L. The relationship between TTF-1 expression and EGFR mutations in lung adenocarcinomas. PLoS One. 2014; 9: e95479.

26. Takeuchi T, Tomida S, Yatabe Y, Kosaka T, Osada H, Yanagisawa K, Mitsudomi T, Takahashi T. Expression profile-defined classification of lung adenocarcinoma shows close relationship with underlying major genetic changes and clinicopathologic behaviors. J Clin Oncol. 2006; 24:1679-1688. 